\title{
194 Pathology of nasal polyps
}

A Allergic polyps are usually bilateral.

B Epithelial metaplasia occurs in polyps of vasomotor rhinitis.

C Fibrosis obstructing lymphatic drainage has a role in pathogenesis.

D Malignant polyps are usually indistinguishable from benign clinically.

E Childhood nasal glioma is a malignant polyp.

\section{Antrochoanal polyps}

A Commonest in the elderly.

B Usually multiple.

C Unilateral nasal obstruction is the commonest symptom.

D The maxillary sinus is opaque on $X$-rays.

$E$ Most can be completely removed intranasally.

\section{Treatment of simple nasal polyps}

A Beclomethasone nasal drops will shrink some polyps.

B Systemic steroids are occasionally used in severe cases.

C Antihistamines may improve rhinorrhoea and sneezing.

D Simple snaring of polyps is no longer performed because of the high incidence of recurrence.

E Intranasal ethmoidectomy is the initial treatment of choice.

\section{Epiphora}

A is excessive watering of the eye due to nasolacrimal duct or sac obstruction.

B Congenital atresia is the commonest cause.

C May be due to a malignant tumour of the maxillary antrum.

D Initial treatment is by probing the nasolacrimal duct.

E Dacrocystorhinostomy aims to divert tears to the opposite nostril via a plastic tube passed through the septum. 